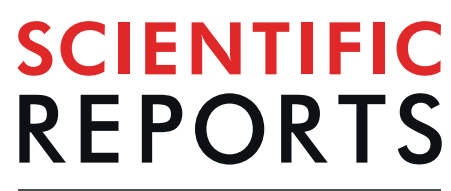

\title{
OPEN The C-Fern (Ceratopteris richardii) genome: insights into plant genome evolution with the first partial homosporous fern genome assembly
}

\author{
D. Blaine Marchant ${ }^{1,2,3^{*}}$, Emily B. Sessa ${ }^{2,4}$, Paul G. Wolf ${ }^{5,6}$, Kweon Heo ${ }^{7}$, W. Brad Barbazuk ${ }^{2,4}$, \\ Pamela S. Soltis $\mathbb{1}^{3,4,8}$ \& Douglas E. Soltis $2,3,4,8$
}

Ferns are notorious for possessing large genomes and numerous chromosomes. Despite decades of speculation, the processes underlying the expansive genomes of ferns are unclear, largely due to the absence of a sequenced homosporous fern genome. The lack of this crucial resource has not only hindered investigations of evolutionary processes responsible for the unusual genome characteristics of homosporous ferns, but also impeded synthesis of genome evolution across land plants. Here, we used the model fern species Ceratopteris richardii to address the processes (e.g., polyploidy, spread of repeat elements) by which the large genomes and high chromosome numbers typical of homosporous ferns may have evolved and have been maintained. We directly compared repeat compositions in species spanning the green plant tree of life and a diversity of genome sizes, as well as both short- and longread-based assemblies of Ceratopteris. We found evidence consistent with a single ancient polyploidy event in the evolutionary history of Ceratopteris based on both genomic and cytogenetic data, and on repeat proportions similar to those found in large flowering plant genomes. This study provides a major stepping-stone in the understanding of land plant evolutionary genomics by providing the first homosporous fern reference genome, as well as insights into the processes underlying the formation of these massive genomes.

There are estimated to be over 400,000 species of extant land plants ${ }^{1}$, encompassing an enormous array of morphological, physiological, and ecological diversity. Accompanying this diversity is extraordinary variation in genome size ${ }^{2,3}$, spanning a 2,500-fold range from the bladderwort Genlisea aurea $(\sim 60 \mathrm{Mbp})^{4}$ to that of the monocot Paris japonica $(150 \mathrm{Gbp})^{5}$. How these genomes are chromosomally partitioned also varies immensely, as land plants span a 360-fold range in chromosome number, from $2 n=4$ in Haplopappus gracilis, Brachychome dichromosomatica, Zingeria biebersteiniana, and Colpodium versicola to $2 n=1,440$ in the fern Ophioglossum reticulatum, the highest number reported for any eukaryote ${ }^{3,6,7}$. Understanding the processes underlying this enormous breadth in both genome size and chromosome number has long been a major area of interest among evolutionary biologists. However, sampling biases towards smaller, less complex genomes (e.g., Arabidopsis: $135 \mathrm{Mbp}, n=5$ ) and crops have pervaded plant genome projects. Fortunately, recent technological advances have enabled the assembly and analysis of large genomes, such as those of conifers ${ }^{8-10}$, providing novel insights into the processes underlying genome and chromosomal composition. Despite this progress, the large genomes typical of homosporous ferns remain uninvestigated.

${ }^{1}$ Present address: Department of Biology, Stanford University, Stanford, CA, 94305, USA. ${ }^{2}$ Department of Biology, University of Florida, Gainesville, FL, 32611, USA. ${ }^{3}$ Florida Museum of Natural History, University of Florida, Gainesville, FL, 32611, USA. ${ }^{4}$ The Genetics Institute, University of Florida, Gainesville, FL, 32611, USA. ${ }^{5}$ Department of Biology, Utah State University, Logan, UT, 84322, USA. ${ }^{6}$ Present address: Department of Biological Sciences, University of Alabama in Huntsville, Huntsville, AL, 35899, USA. ${ }^{7}$ Department of Applied Plant Sciences, Kangwon National University, Chuncheon, 24341, Korea. ${ }^{8}$ The Biodiversity Institute, University of Florida, Gainesville, FL, 32611, USA. *email: dbmarchant@stanford.edu 
Polyploidy, or whole-genome duplication (WGD), is the traditional explanation for the large genomes and numerous chromosomes found in many plants, as WGD results in the complete doubling of the genome $\mathrm{e}^{11,12}$. Among flowering plants, phylogenetic and genomic studies have identified WGD events preceding key radiations, such as those of the core eudicots $(\sim 70 \% \text { of the flowering plants })^{13}$, monocots ${ }^{14}$, and the entirety of flowering plants ${ }^{15-17}$. In addition, it was demonstrated that even species with minute genomes, such as the carnivorous plant Utricularia gibba, with $n=13$ and a genome size of $80 \mathrm{Mbp}$, have experienced multiple WGD events; $U$. gibba has undergone at least three ancient WGD events in the last 80 million years ${ }^{18}$. Thus, genome size and chromosome number may not be reliable indicators of WGD. This disparity between genome size and chromosomal composition relative to WGD frequency has altered our understanding of genome evolution as the question has changed from whether or not an organism is polyploid, to how many rounds of polyploidy an organism or lineage has experienced in its evolutionary history.

Although a few fern genomes, such as those of the heterosporous water ferns ( $<1 \%$ of fern diversity), are less than $250 \mathrm{Mbp}^{19}$, the average homosporous fern genome is $12 \mathrm{Gbp}$, nearly five times the size of the genome of maize (2.5 Gbp) and over 80 times that of Arabidopsis. In addition, homosporous ferns typically have substantially more chromosomes than seed plants, with an average haploid chromosome number of 59 compared to 16 in flowering plants or 12 in gymnosperms ${ }^{3,20}$. As a result, longstanding hypotheses have proposed that multiple, repeated WGD events were the major factor contributing to the high chromosome numbers and large genomes of ferns ${ }^{20-22}$.

It was originally hypothesized that homosporous ferns undergo intense selection favoring polyploidy to buffer against a putatively high rate of inbreeding that results from their unique life history ${ }^{20,23}$. The homosporous fern life cycle includes a free-living haploid gametophyte phase with the potential for intragametophytic selfing $(\mathrm{IGS})^{23}$, or gametophytic selfing sensu Haufler et al. ${ }^{24}$ - a process that can produce a completely homozygous diploid plant in a single generation and thus expose any deleterious mutations. However, numerous isozyme analyses demonstrated that fern species with the lowest chromosome numbers within a given genus (ranging from $n=27$ to 52 ) were functionally diploid, producing typical diploid numbers of isozyme loci rather than multiple loci as seen in truly polyploid species with multiples of these low chromosome numbers ${ }^{25-27}$. Despite the lack of isozyme evidence for repeated polyploidy in diploid fern species, multiple copies of chlorophyll a/b-binding protein genes were discovered in the diploid fern Polystichum munitum, but the duplicated genes were nonfunctional $^{28}$. Furthermore, early population genetic investigations showed that homosporous ferns have highly variable mating systems and are typically outcrossing, refuting the hypothesized force (intense inbreeding depression via IGS) driving selection for polyploidy ${ }^{29}$. More recently, a genetic linkage map showed that Ceratopteris richardii has one of the highest proportions of duplicated loci among plants (76\%) yet lacks large, duplicated blocks that would be indicative of polyploidy ${ }^{30}$. In addition, a paralog-age distribution analysis of Ceratopteris estimated an ancient polyploidy event over 180 million years ago (mya); however, the data used for this analysis were from a shallow EST library ${ }^{31}$. Despite little evidence for ancient polyploidy in ferns, chromosome count models suggest that $31 \%$ of fern and lycophyte speciation events involve WGD, compared to $15 \%$ in flowering plants ${ }^{32}$. However, these estimates of WGD refer to relatively recent polyploidy events (neopolyploidy) evident from chromosome numbers rather than ancient (paleopolyploidy) events deep in evolutionary history.

Ferns are also the only major lineage of land plants with a significant positive relationship between genome size and chromosome number, suggesting that fern chromosomes are relatively static compared to those of angiosperms and gymnosperms for which no such correlation exists ${ }^{33,34}$. While repeated episodes of WGD followed by extensive silencing and rearrangement cannot be discounted as an explanation for the paradoxical genomic, genetic, and chromosomal composition of ferns ${ }^{21}$, alternative processes underlying their large genomes and high chromosome numbers must be explored. Most notable among these alternative explanations for the large genomes of ferns is the impact of transposable elements (TEs) on genome size, as TEs make up the majority of genome space in a variety of eukaryotic lineages. For example, TEs are responsible for the difference in genome size between cultivated rice (Oryza sativa, $390 \mathrm{Mbp}$ ) and wild rice (O. australiensis, $965 \mathrm{Mbp})^{35}$. Phylogenetic reconstructions of major TE families in various plant lineages suggest that bursts of TE insertion result in inflated genome size $\mathrm{s}^{36-39}$. However, genome inflation does not seem to be a one-way street, because unequal homologous recombination can eliminate repetitive regions, such as those produced by $\mathrm{TEs}^{40,41}$. Analysis of three conifer "giga-genomes" (20-30 Gbp) showed that these large genomes were derived not through WGD, but rather via extensive expansion of ancient TEs (especially retrotransposons) and an apparent inability to shed these repetitive regions via unequal recombination ${ }^{8,42}$. While TEs provide a possible alternative explanation for the large genome sizes of ferns as demonstrated in conifers, they cannot explain the high chromosome numbers of ferns. It is possible that ferns have ancestrally high chromosome numbers and a relatively low rate of WGD, yet this begs the question of how the high chromosome numbers were initially obtained. Aneuploidy or chromosomal fission are also possible explanations for the high chromosomal complement of most ferns ${ }^{22,26,43}$.

There are now hundreds of published flowering plant, gymnosperm, lycophyte, and bryophyte genomes, alongside the recent publication of two heterosporous water fern genomes ${ }^{19}$. While these water fern genomes, for Azolla and Salvinia, are much-needed references within the fern clade, they are atypical of $99 \%$ of ferns, in that these species are heterosporous and have very small genomes with few chromosomes $(1 \mathrm{C}=0.25-1.76 \mathrm{Gbp}$, $n=9-22)^{19}$. To date, no sequenced genome is yet available for any homosporous fern ${ }^{44}$. This major information gap is made more startling when the high species diversity ( $>10,000$ species), significant ecological roles, and economic importance of homosporous ferns are considered ${ }^{45-50}$. Due to their crucial phylogenetic position as sister to seed plants, ferns are key for investigating an array of both genomic and non-genomic traits and will permit a synthesis of genome evolution across seed plants ${ }^{51,52}$.

Here we investigated the genome of the homosporous fern Ceratopteris richardii (C-fern; $11.25 \mathrm{Gbp}, n=39$ ), characterizing and classifying TE composition and assessing the extent of WGD. Our genomic data for C-fern, together with the recently published heterosporous water fern genomes ${ }^{19}$, help provide a genome evolutionary 


\begin{tabular}{|c|c|}
\hline Cytometric Genome Size & $11.25 \mathrm{Gbp}$ \\
\hline Chromosome number & 39 \\
\hline \multicolumn{2}{|l|}{ Assembly V 1.0} \\
\hline Meraculous Contigs & $15,871,274$ contigs \\
\hline Total Size & $4.21 \mathrm{Gbp}$ \\
\hline N50\% & $300 \mathrm{bp}$ \\
\hline Gaps & 0 \\
\hline$\% \mathrm{GC}$ & 36 \\
\hline CFern $v 1.0(\geq 1,000 \mathrm{bp})$ & 988,403 scaffolds \\
\hline Total Size & $2.69 \mathrm{Gbp}$ \\
\hline N50 & $3,376 \mathrm{bp}$ \\
\hline$\%$ Gaps & 0.5 \\
\hline$\% \mathrm{GC}$ & 36 \\
\hline CFern $v 1.1(\geq 1,000 \mathrm{bp})$ & 626,576 scaffolds \\
\hline Total Size & $4.25 \mathrm{Gbp}$ \\
\hline N50 & $16,289 \mathrm{bp}$ \\
\hline$\%$ Gaps & 37 \\
\hline$\% \mathrm{GC}$ & 38 \\
\hline CFern v1.1A $(\geq 10,000 \mathrm{bp})$ & 133,755 scaffolds \\
\hline Total Size & $2.79 \mathrm{Gbp}$ \\
\hline N50 & $22,401 \mathrm{bp}$ \\
\hline$\%$ Gaps & 44 \\
\hline$\% \mathrm{GC}$ & 38 \\
\hline BAC.SubSample & 35 scaffolds \\
\hline Total Size & $3.03 \mathrm{Mbp}$ \\
\hline N50 & $97,182 \mathrm{bp}$ \\
\hline$\%$ Gaps & 0 \\
\hline$\% \mathrm{GC}$ & 39 \\
\hline
\end{tabular}

Table 1. Ceratopteris genome assembly statistics.

context not just for ferns, but also for all vascular plants. Collectively, these data will permit deductions about ancestral genome characteristics of seed plants and ferns, as in studies of other phylogenetically pivotal lineages $^{16,53}$. Specifically, this Ceratopteris genome provides critical insights into the evolutionary genomics and paradoxes of the genomically long-neglected fern clade, in addition to serving as a valuable reference for future investigations into land plant genome composition and dynamics.

\section{Results}

Genome sequencing and assembly. Here we present the first sequence of a homosporous fern genome, providing a new resource for plant and evolutionary biology. The ability of homosporous ferns to undergo IGS (see above) partially simplified the assembly of this complex genome, as it made the sporophyte completely homozygous so that heterozygosity was not an issue in assembly. However, the quality of the Ceratopteris genome assembly and the computational resources required to assemble and analyze the genome reflect the technological difficulties of working with such a large and complicated genome with no closely related reference genome.

With paired-end short-read libraries totaling $\sim 24 \mathrm{X}$ coverage from 1.8 billion cleaned reads, we assembled the 11.25 Gbp Ceratopteris genome into $\sim 15$ million contigs ( $>100 \mathrm{bp}$ ) or 988,403 scaffolds $(>1,000 \mathrm{bp}$ ) (Table 1$)$. We then combined and reduced the number of scaffolds using 8-10 Kbp mate-pair reads (13X coverage), producing a genome assembly (CFern v1.1) of 626,576 scaffolds with an N50 of $16 \mathrm{Kbp}$ and total length of $4.25 \mathrm{Gbp}$, representing about $38 \%$ of the Ceratopteris genome.

We also sampled a smaller portion of the Ceratopteris genome using long-read sequencing of 32 bacterial artificial chromosomes (BACs) of Ceratopteris. This subsample assembly (BAC.SubSample) only totaled 3 Mbp of the Ceratopteris genome $(0.03 \%)$, but had an $\mathrm{N} 50$ of $97 \mathrm{Kbp}$, providing a small, but more accurate and contiguous sampling of the $11.25 \mathrm{Gbp}$ genome as long-read technology is less biased by repeat elements and mis-assemblies. The GC content of Ceratopteris was $37.7 \%$, very similar to that of both the gymnosperm Picea abies (Norway spruce) (37.6\%) and the flowering plant Amborella trichocarpa (37.5\%), yet lower than that of maize (46.9\%), the liverwort Marchantia polymorpha (42.0\%), and the lycophyte Selaginella moellendorffi (45.3\%).

Transcriptome assembly. From 12 PacBio SMRT cells, we obtained $\sim 850,000$ reads from which we produced 97,084 full-length, high-quality, cleaned transcripts (IsoSeq.HQ) ranging from 285 to 11,353 bp in length. When mapped onto the CFern $v 1.1$ assembly at $98 \%$ identity and $98 \%$ coverage, the IsoSeq.HQ transcripts were collapsed into 4,620 genes and 10,043 isoforms; however, when coverage was reduced to $50 \%$, there were 11,924 genes and 23,278 isoforms. The 2.5-fold increase in identified genes and isoforms via reduced coverage shows that our scaffolds do not span entire genes in the majority of cases. To overcome this fragmentation and provide 


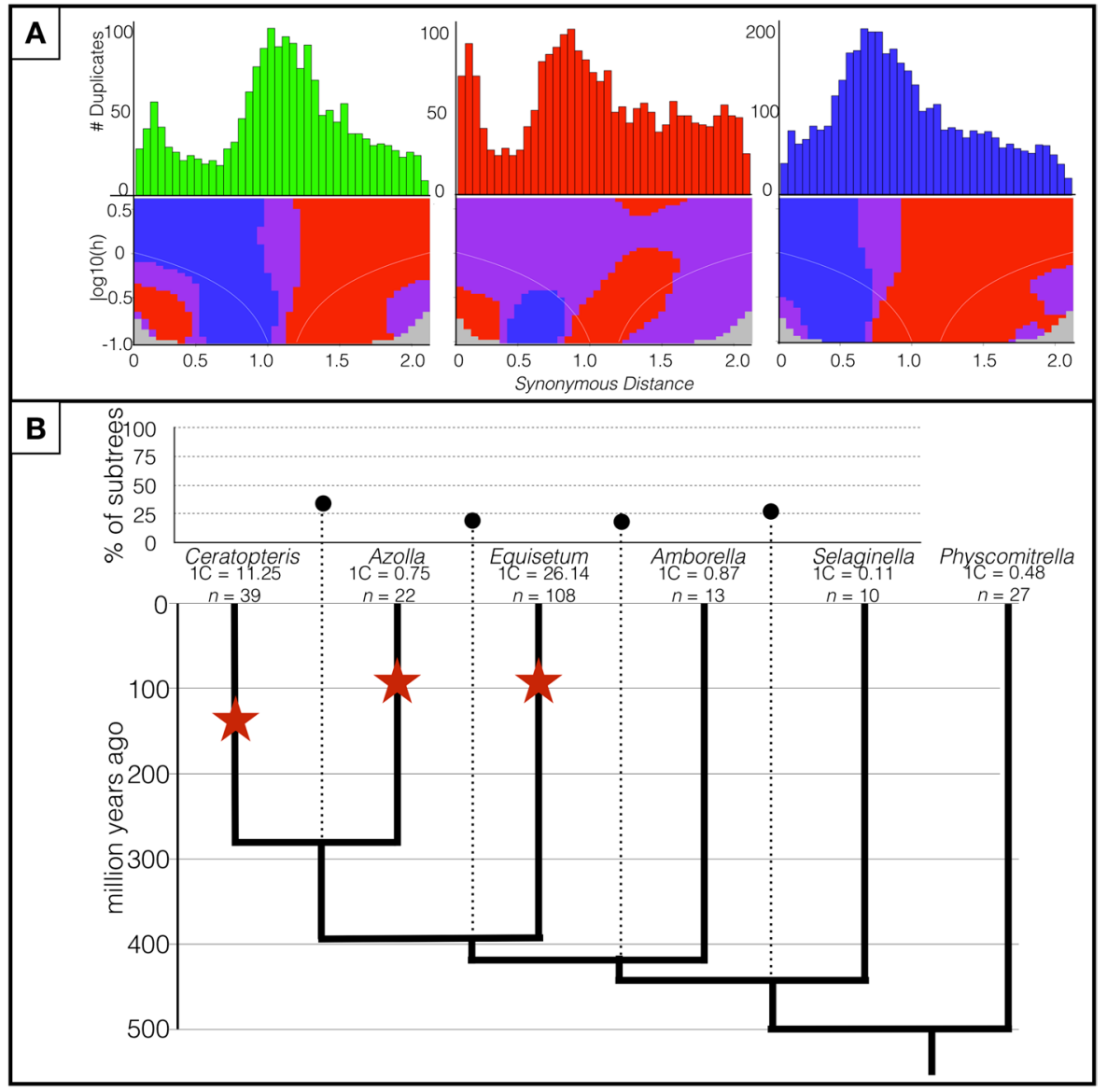

Figure 1. Polyploidy analyses of three fern species. (A) Paralog-age distribution analyses and associated SiZER plots of three fern species. Upper panels are Ks-based histograms ( 0.05 bins) of paralogs in Ceratopteris richardii, Azolla filiculoides, and Equisetum giganteum. Lower panels are SiZER plots of the above paralog-age distribution data and associated smoothing functions where blue indicates significant $(\alpha=0.05)$ increases, red significant decreases, purple insignificance, and gray too few data points to determine. The white lines show the effective window widths for each bandwidth. Both upper and lower panels are on the same $x$-axis. (B) MAPS analysis across land plants and the associated WGD events (shown as stars). The percentages of subtrees that contain gene duplications shared by the descendent species of a given node are above the phylogeny (connected by dotted lines). Dates are based on Testo and Sundue ${ }^{70}$ and Morris et al. ${ }^{67}$.

a set of high-confidence gene models, we implemented the Cogent genome-free protocol $^{54}$ to produce 18,179 gene models (UniCFernModels) from the IsoSeq.HQ transcripts. Searching for 1,440 embryophyte single-copy orthologs ${ }^{55}$, we found $53 \%$ complete, $4.5 \%$ fragmented, and $42.6 \%$ missing.

Polyploidy. To address the decades-old question of how common ancient polyploidy is in ferns, we employed sequence-based and cytogenetic approaches, which assessed three different temporal scopes of evolutionary history. Using paralog-age distribution analyses, we identified 1,800 paralogous gene pairs in the UniCFernModels with a $K_{S}$ value between 0.1 and 2.1. A minor peak around $K_{S}=0.3$ was detected; however, such small, "recent" peaks are often a result of small-scale gene duplications, not $\mathrm{WGD}^{56}$. In contrast, a single major peak was revealed in the synonymous distance plot of Ceratopteris, similar to those observed in Azolla and Equisetum (Fig. 1). Based on the significant transition from positive to negative in the SiZER plot, the Ceratopteris peak was at $K_{S}=1.1$, compared to 0.8 in Azolla and 0.75 in Equisetum, similar to the original results found by Vanneste et al. ${ }^{57}$.

To determine whether the peaks found in these three ferns (Ceratopteris, Azolla, Equisetum) represent a shared WGD rather than three distinct WGD events, we used the Multi-taxon Paleopolyploidy Search (MAPS) ${ }^{58}$. We first recovered 10,182 orthogroups from the clustered amino acid sequences of Ceratopteris, Azolla, Equisetum, Amborella, Selaginella, and Physcomitrella. We isolated 4,836 orthogroups with amino acid sequences from all six species and estimated gene family trees for each orthogroup. Of the subtrees that fit the known fern topology, ((Ceratopteris, Azolla), Equisetum), 34\% supported a gene duplication in the most recent common ancestor (MRCA) of Ceratopteris and Azolla, and 19\% of subtrees fitting the ((Ceratopteris/Azolla, Equisetum), Amborella) topology supported a gene duplication shared across the three fern species (Fig. 1) - relatively low proportions compared to similar studies that identified shared WGD events ${ }^{58}$. These low proportions suggest three lineage-specific WGD events rather than one or two shared events between the three fern taxa. 


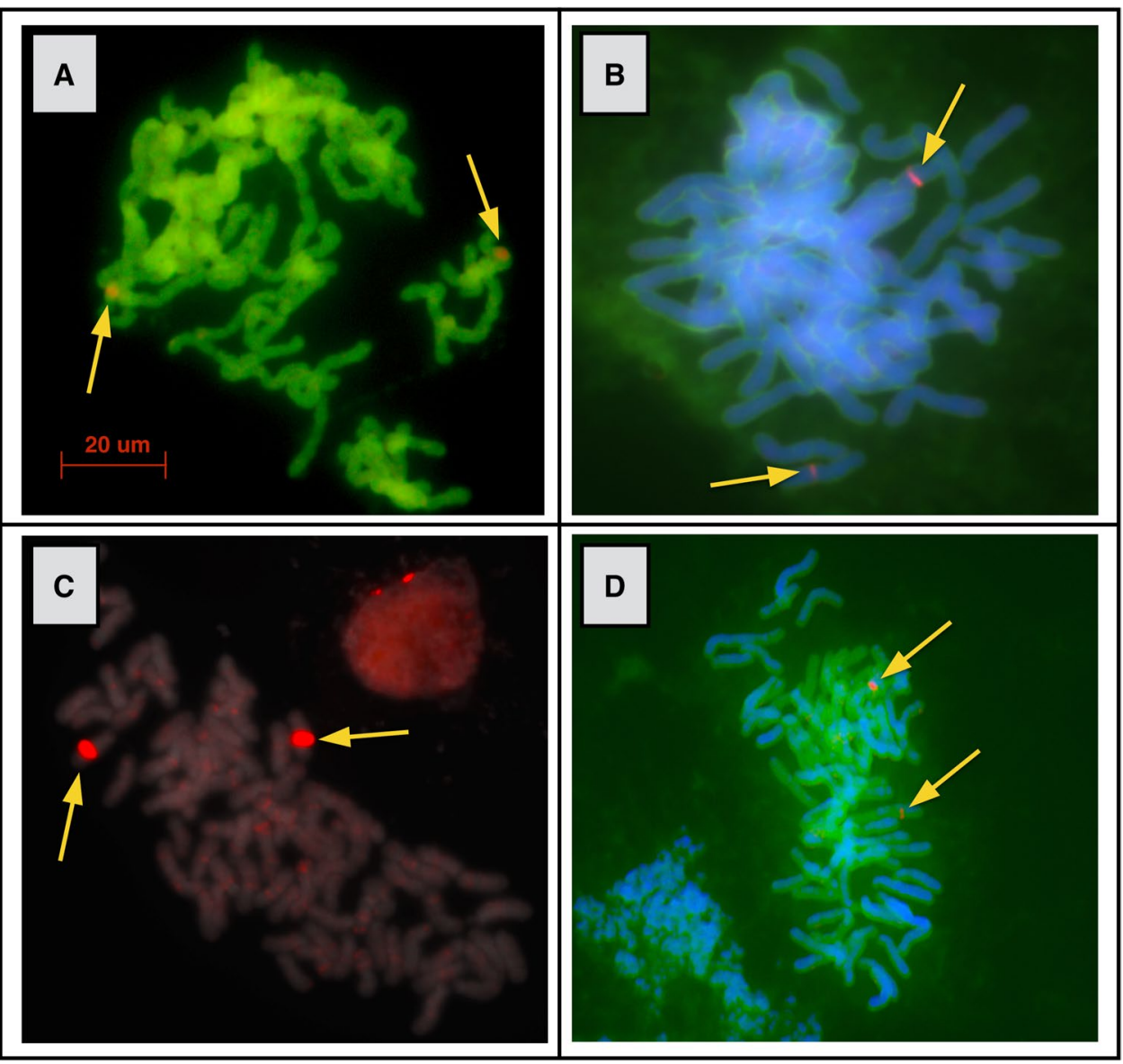

Figure 2. Fluorescent in situ hybridizations of Ceratopteris chromosome spreads. The fluorescent probes are of 100-150 Kbp DNA fragments from BACs of Ceratopteris. Primary "diploid” localizations (red bands labeled with arrows) are shown in all four panels, while weak secondary localizations, most likely reflecting repetitive elements, are apparent in $(\mathbf{C})$; note scattered faint red staining in addition to the two strong primary signals. BACs are from wells A12 (A), B3 (B), A8 (C), and B9 (D) in Plate CR_Ba \#624, Green Plant BAC Library Project, Clemson University Genomics Institute.

While the previously described methods of data analysis for assessing WGD are appropriate at deeper time scales, both are susceptible to missing more recent WGD events. As mentioned above, relatively recent (close to zero along the $x$-axis) WGD in the $K_{S}$ plots may be mistakenly attributed to small-scale duplications, while MAPS can only identify WGD events that have occurred prior to the MRCA of the next closest taxon included in the analysis. In the case of Ceratopteris, that would be 280 million years to the divergence of Ceratopteris and Azolla, thus only events older than that can be identified by MAPS.

Our cytogenetic approach using FISH suggests the ploidy of an organism by localizing 125-150 Kbp BAC DNA fragments to the chromosomes where the DNA fragment is found. If the organism is diploid, only two localizations will be apparent, while a polyploid should have more than two localizations. BAC-FISH evidence of polyploidy is relatively short-lived as studies of Nicotiana allopolyploids found that five million years after the WGD event, the two parental genomes in the polyploid were no longer distinguishable due to genome turnover, mutations, and small-scale duplications ${ }^{59}$. However, our BAC-FISH results further corroborated our sequencing results in demonstrating a lack of evidence for recent WGD in Ceratopteris. Significantly, we detected only two primary localizations of each BAC probe we exposed to the Ceratopteris chromosome preparations (Fig. 2), suggesting diploidy. In a few cases, weak secondary localizations, or "ghost bands," were found on multiple chromosomes; however, these are likely a result of repeat elements that are distributed throughout the numerous chromosomes.

Repeat diversity. In total, $\sim 42 \%$ of the CFern $v 1.1 \mathrm{~A}$ assembly was composed of repeat elements (Fig. 3a). The Copia LTR RTs were the most prolific with over 800,000 elements making up $16.5 \%$ of the assembled genome, followed by the Gypsy LTR RT superfamily with 330,000 elements and accounting for 7.5\% of the genome (Table 2). In comparison, Class II DNA transposons include members of 17 different super-families, yet only totaled 52,000 elements and $<1 \%$ of the genome. The LINE RTs similarly covered $1.6 \%$ of the genome across 64,000 elements. Low-complexity, satellite, and simple repeats all covered $<0.5 \%$ of the genome.

The repeat content and percent coverage were considerably higher in the long-read BAC.SubSample assembly (63\%). Nearly $26 \%$ of the subsample assembly was made up of Gypsy LTR RTs and $21.8 \%$ was Copia LTR RTs, 


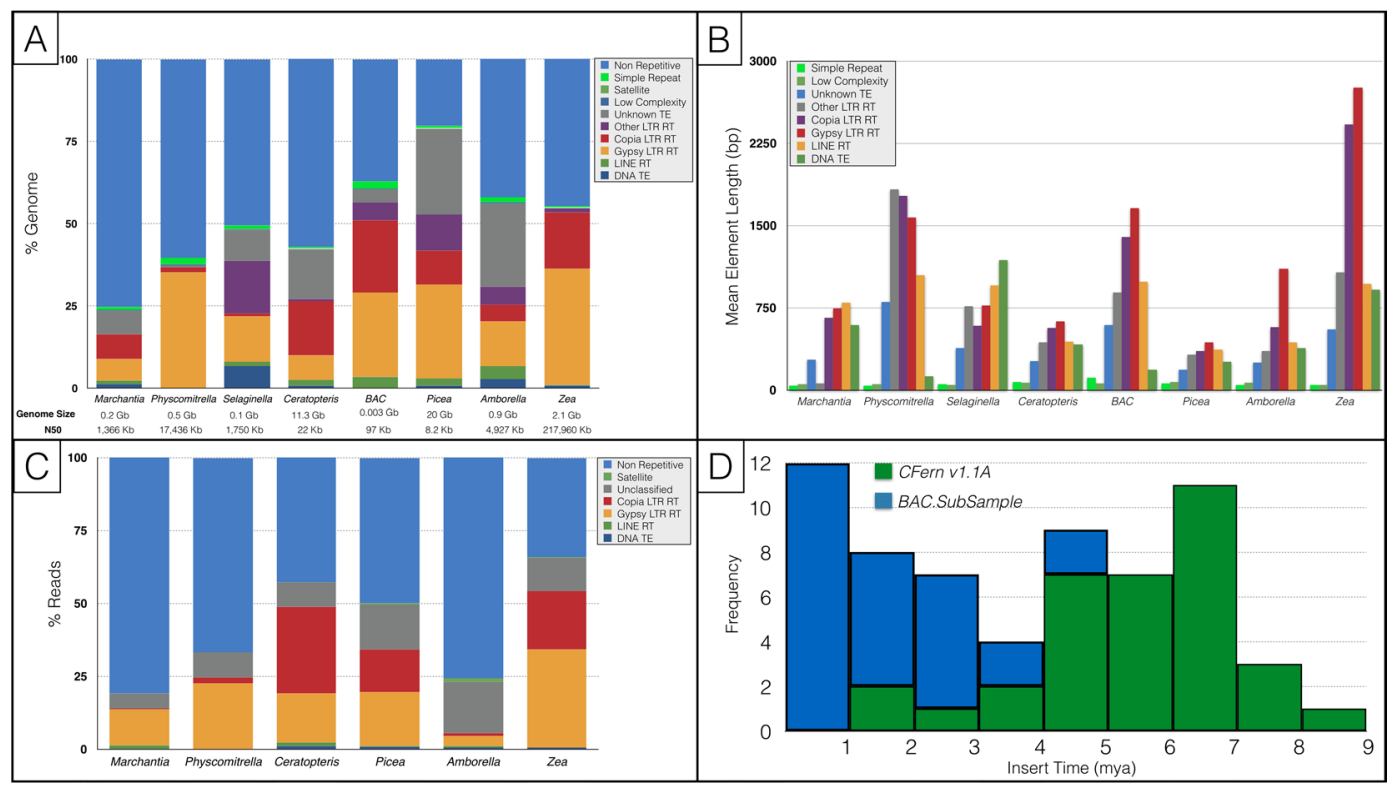

Figure 3. Repeat composition, lengths, and insertion timing for representative embryophyte genome assemblies. (A) Genome proportions of repetitive and non-repetitive elements for seven taxa spanning land plants, as well as BAC.SubSample, using genome-based analyses. Genome sizes and N50s for analyzed genome assemblies are also provided. (B) Mean repeat element lengths based on genome assembly analyses (A) for seven embryophyte taxa and BAC.SubSample. (C) Genome proportion of repetitive and non-repetitive elements using read-based clustering analyses ${ }^{111}$. (D) LTR RT insertion dates in Ceratopteris based on the CFern v1.1A and BAC.SubSample assemblies. Insertion dates were inferred from the similarity of long terminal repeat regions of the LTR RTs and a neutral substitution rate of $6.5 \times 10^{-9}$ per site per year.

while the LINE RTs and DNA transposons represented 3.2\% and $0.16 \%$, respectively. Low-complexity and simple repeats made up $0.2 \%$ and $2.2 \%$ of the BAC.SubSample, respectively. The mean lengths of all of the repeat types in the BAC.SubSample, with the exception of the DNA TEs, were more than double those of the CFern v1.1 A assembly, and the Copia and Gypsy elements were nearly three times as large in the subsample compared to those of the CFern $v 1.1 \mathrm{~A}$ assembly.

Similar to the BAC.SubSample results, read-based analyses of Ceratopteris also estimated that $\sim 60 \%$ of the Ceratopteris genome is repetitive with 17\% in Gypsy elements and 30\% in Copia elements (Fig. 3c). The read-based analysis and BAC.SubSample characterization analysis are more accurate for assessing general genome composition compared to repeat characterization of the CFern $v 1.1 \mathrm{~A}$ assembly, as they are not biased by the short-read assembly which can have trouble assembling repetitive regions beyond the length of the reads. This assembly bias is probably the reason the CFern $v 1.1$ assembly was limited in low-complexity, satellite, and simple repeat elements. This limitation is also apparent in comparing the mean lengths of the CFern $v 1.1 \mathrm{~A}$ LTR RTs to those of the BAC.SubSample (Fig. 3b), as the latter assembly could likely span those longer repetitive regions via long-read technology.

We directly compared the repeat content of Ceratopteris to that of other land plants by applying the same assembly-based repeat characterization protocol to Amborella trichopoda ${ }^{16}$, a monocot (Zea mays $)^{60}$, a liverwort (Marchantia polymorpha $)^{61}$, a lycophyte (Selaginella moellendorffii) ${ }^{62}$, a conifer (Picea abies) ${ }^{8}$, and a moss (Physcomitrella patens) ${ }^{63}$. We chose to run our own analyses on these genome assemblies rather than comparing our results to those of previously published results due to the wide variation in repeat characterization analyses utilized. In addition, we ran the read-based clustering analysis on the above taxa, with the exception of Selaginella, using short reads from these assemblies covering $0.5 \mathrm{X}$ of each genome. Due to the previously mentioned limitations of the CFern v1.1 A assembly, we largely focused on the BAC.SubSample and read-based results for comparing relative proportions of repeats to other taxa.

Compared to six other land plant genome assemblies, the BAC.SubSample was second in repeat proportion behind only Picea (Fig. 3a). The BAC.SubSample had proportions of Copia elements similar to those of Zea, substantially higher than any of the other genomes analyzed. The other super-family of LTR RTs, the Gypsy elements, represented $25.7 \%$ of the BAC.SubSample with a mean length of $1,660 \mathrm{bp}$. In contrast, $35 \%$ of the Zea and Physcomitrella genomes were made up of Gypsy elements with mean lengths of 2,755 and 1,574bp, respectively. The read-based analyses generally agreed with the repeat proportions of the six analyzed taxa with the exceptions of Amborella and Picea (Fig. 3c). These two taxa had lower overall repeat proportions in the read-based analyses (58\% vs. $25 \%$ in Amborella, $80 \%$ vs. $50 \%$ in Picea), matching previous similar studies ${ }^{64}$.

We assessed LTR RT richness by comparing recent (>90\% LTR similarity) LTR RT exemplars among the seven species compared here (Table 3). Zea was by far the most diverse with 4,561 distinct LTR RT exemplars, followed by Physcomitrella at 1,217 exemplars and Picea with 509. The CFern v1.1 A assembly was low in recent 


\begin{tabular}{|c|c|c|c|c|c|}
\hline Class & Order & Superfamily & \begin{tabular}{|l}
$\begin{array}{l}\text { Element } \\
\text { count }\end{array}$ \\
\end{tabular} & Length (bp) & $\%$ Genome \\
\hline \multirow{15}{*}{ Retrotransposon } & \multirow{8}{*}{ LINE } & Uncategorized & 1458 & 523887 & 0.02 \\
\hline & & RTE-BovB & 421 & 128815 & 0.00 \\
\hline & & Jockey & 434 & 127827 & 0.00 \\
\hline & & R1 & 2565 & 1241869 & 0.04 \\
\hline & & RTE-X & 8733 & 3937956 & 0.14 \\
\hline & & L2 & 19047 & 3652063 & 0.13 \\
\hline & & L1-Tx1 & 23635 & 15522256 & 0.56 \\
\hline & & L1 & 47105 & 20464731 & 0.73 \\
\hline & \multirow{7}{*}{ LTR } & Uncategorized & 23507 & 7859618 & 0.28 \\
\hline & & DIRS & 361 & 45453 & 0.00 \\
\hline & & Pao & 1494 & 462814 & 0.02 \\
\hline & & Gypsy-Troyka & 5331 & 3393861 & 0.12 \\
\hline & & ERV1 & 8083 & 6191165 & 0.22 \\
\hline & & Gypsy & 329706 & 207014935 & 7.42 \\
\hline & & Copia & 812470 & 460237954 & 16.50 \\
\hline \multirow{18}{*}{ DNA Transposon } & & Uncategorized & 3289 & 693293 & 0.02 \\
\hline & & hAT-Tip100 & 374 & 251768 & 0.01 \\
\hline & & CMC-Mirage & 416 & 153347 & 0.01 \\
\hline & & MULE-MuDR & 425 & 41133 & 0.00 \\
\hline & & TcMar & 530 & 83430 & 0.00 \\
\hline & & hAT-hATw & 622 & 343180 & 0.01 \\
\hline & & Harbinger & 627 & 224160 & 0.01 \\
\hline & & \begin{tabular}{|l|} 
PiggyBac \\
\end{tabular} & 1230 & 122417 & 0.00 \\
\hline & & Dada & 1981 & 1130399 & 0.04 \\
\hline & & CMC-EnSpm & 2339 & 1276711 & 0.05 \\
\hline & & Sola & 2739 & 1565090 & 0.06 \\
\hline & & hAT & 2774 & 819873 & 0.03 \\
\hline & & \begin{tabular}{|l|} 
Maverick \\
\end{tabular} & 4082 & 2443709 & 0.09 \\
\hline & & hAT-Ac & 4625 & 1727566 & 0.06 \\
\hline & & PIF-Harbinger & 4982 & 1214259 & 0.04 \\
\hline & & En-Spm & 10115 & 5487072 & 0.20 \\
\hline & & hAT-Tag1 & 15720 & 6076071 & 0.22 \\
\hline & & Helitron & 4260 & 812693 & 0.03 \\
\hline
\end{tabular}

Table 2. Ceratopteris repeat diversity and composition.

\begin{tabular}{|l|l|l|l|l|l|l|l|}
\hline & Selaginella & Marchantia & Physcomitrella & Amborella & Zea & Ceratopteris & Picea \\
\hline Genome Size & 0.11 & 0.2 & 0.48 & 0.87 & 2.1 & 11.25 & 20 \\
\hline \% GC & 45.3 & 42 & 33.7 & 37.5 & 46.9 & 37.7 & 37.6 \\
\hline N50 (Kbp) & 1750 & 1366 & 17435 & 4927 & 217959 & 22 & 8 \\
\hline Recent LTR RT & 166 & 30 & 1217 & 11 & 4561 & 22 & 509 \\
\hline Ancient LTR RT & 33 & 24 & 16 & 55 & 45 & 82 & 276 \\
\hline
\end{tabular}

Table 3. Genome composition and LTR-RT statistics in sampled land plant genomes.

LTR RT diversity with only 22 exemplars, similar to that of Amborella and Marchantia, which had 11 and 30 , respectively. Ancient (75-90\% LTR similarity) LTR RT richness differed greatly from recent LTR RT diversity in Zea and Physcomitrella, which only had 45 and 16 ancient exemplars, respectively (Table 3). Picea also had fewer ancient exemplars than recent LTR RTs with 276, but CFern v1.1 A and Amborella both had more ancient than recent LTR RT exemplars with 82 and 55 , respectively.

The quality of the genome assembly could have had a large effect on these interspecific comparisons of repeat diversity, number, and size, as well as genome size, as earlier demonstrated with the BAC.SubSample. For example, these eight genome assemblies spanned a 27,000-fold difference in scaffold N50 lengths between that of Picea (8 $\mathrm{Kbp})$ and maize $(217,960 \mathrm{Kbp})$. Thus, in addition to the genomes of CFern $v 1.1 \mathrm{~A}$ and Picea being many times larger than that of maize, they are much more fragmented, making the identification of repeat elements more difficult and biased for identifying those repeats with smaller lengths.

To investigate LTR RT insertion timing, we identified 62 full-length, high-confidence LTR RT elements in the CFern v1.1 A and BAC.SubSample assemblies ${ }^{65}$. The insertion timing of these LTR RTs was relatively uniform 
over the past 7 million years (Fig. 3d). However, we found considerable differences in the LTR RT identification of these two assemblies as the majority of the identified LTR RTs in the BAC.SubSample originated within the last million years, while the CFern $v 1.1 A$ assembly did not identify a single LTR RT within the past million years and instead had largely older ( $>4$ mya) LTR RTs. In addition, we note that the BAC.SubSample had 28 full-length, high-confidence LTR RTs, while the CFern $v 1.1 \mathrm{~A}$ assembly had 34, despite nearly a 1,000-fold difference in assembly length. These results suggest that the long-read sequencing of the BAC.SubSample was able to span and properly assemble these repetitive elements, while the short reads of CFern $v 1.1$ could only assemble older, more heterogeneous repetitive elements.

\section{Discussion}

Ferns are the second most species-rich clade of vascular plants, with over 10,000 species ${ }^{50,66}$. In addition, ferns are the sister lineage to all seed plants and first appeared approximately 430 million years ago according to fossil-calibrated phylogenies ${ }^{67}$. The oldest unequivocal fossil fern is Ibyka amphikoma with a minimum age of 384 million years ${ }^{67,68}$. However, most extant fern diversity arose within the last 40-60 million years during the Cenozoic Era following the rise to dominance of the angiosperms ${ }^{69,70}$. Despite their substantial morphological diversity, sister relationship to seed plants, and lengthy evolutionary history, ferns represent the final frontier of land plant genomics.

Here we provide the first draft genome assembly of the 11.25 Gbp Ceratopteris genome, as well as a high-confidence set of gene models. We assessed the role of WGD in the evolutionary history of Ceratopteris at three distinct temporal scales. Despite a genome size five times that of classically "large-genome" flowering plants (e.g., maize) and with eight times more chromosomes than Arabidopsis, which has undergone at least five WGD events ${ }^{16}$, we found evidence consistent with only one ancient WGD event in Ceratopteris. The diploid signal localizations of our BAC-FISH approach refute any recent WGD events that may have been ambiguous in the paralog-age distribution analyses. The three peaks in the paralog-age distribution analyses of Ceratopteris, Azolla, and Equisetum overlap and thus could potentially be a shared event before the divergence of these three ferns (Fig. 1). However, MAPS analysis indicates that only a minority of subtrees support shared duplications among these three taxa, suggesting three lineage-specific WGD events rather than a single shared event. Based on our analyses and the timing of the WGD event in Equisetum, another lineage belonging to the broader fern (monilophyte) clade ${ }^{57}$, the WGD of Ceratopteris is likely older than that of Equisetum (92 mya) yet younger than the most recent common ancestor of Ceratopteris and Azolla $(\sim 280 \mathrm{mya})^{70}$ (Fig. 1b). The approaches used here are those standardly used for inferring ancient WGD events from transcriptomic and genomic data. Even complete transcriptomes or chromosome-level genomes can underestimate the true number of WGDs as pseudogenes and gene fragments may be filtered out during analyses. As such, all paralog-age distribution analyses and the resulting inferences regarding WGDs are considered minimum estimates; however, similar analyses of diploid and triploid Ceratopteris thalictroides found nearly identical $K s$ frequency distributions to the results presented in this study ${ }^{71}$. If additional WGD events were obscured from the Ks analyses due to incomplete gene sampling, it is unlikely that transcriptome samples from a congeneric species would hide the same events. The authors of the above-mentioned study dated the WGD event to $52 \pm 1$ mya due to their use of a relatively rapid synonymous substitution rate $\left(11.04 \times 10^{-9}\right)^{71}$. Synonymous substitution rates are highly variable among plant lineages and across time $\mathrm{e}^{72,73}$; thus, we are more confident in our relative dating of the Ceratopteris WGD as occurring between that of Equisetum and the most recent common ancestor of Ceratopteris and Azolla due to our MAPS results.

Our results do not support hypotheses of frequent WGD in ferns followed by massive gene silencing and the slow loss of genetic material ${ }^{74,75}$ and instead lend credence to the hypothesis that ferns had ancestrally high chromosome numbers ${ }^{26,43}$ and underwent WGD rarely yet were unable or very slow to lose genetic material ${ }^{21,31}$. This conclusion is in agreement with past studies based on isozymes ${ }^{25-27}$, transcripts of Equisetum giganteum ${ }^{57}$, a genetic linkage map of Ceratopteris ${ }^{30}$, as well as ancestral reconstructions ${ }^{76}$ that found ancient WGD events to be rare in the evolutionary history of ferns despite the presence of many neopolyploids ${ }^{32}$. While genomic analyses in flowering plants have shown that even very small genomes, such as that of Arabidopsis, have undergone numerous rounds of polyploidy, yet still have a low number of chromosomes, we find that ferns are much less dynamic, having undergone relatively few WGD events, yet retaining a high number of chromosomes. Ancestral reconstructions of chromosome numbers across ferns have suggested that the common ancestor of all ferns had a haploid chromosome number of 22 , while many of the more diverse fern lineages had higher ancestral chromosome numbers, such as $n=30$ in Pteridaceae ${ }^{76}$. If chromosome numbers were ancestrally high in ferns, only a single WGD event would therefore be needed to reach a chromosome number of $n=39$ in Ceratopteris (or $n=59$, the average across all ferns) since the divergence of the common ancestor of ferns from that of seed plants 400 million years ago ${ }^{67,70}$. Alternatively, the high chromosome numbers of ferns could be a result of aneuploidy or chromosomal fission ${ }^{42}$. To better understand the cause of the high chromosome numbers of homosporous ferns, comparative syntenic and phylogenomic analyses will have to be applied across multiple fern taxa based on complete genome assemblies.

Similar to other large plant genomes, a large proportion of the Ceratopteris genome is composed of LTR RTs and other transposable elements (Fig. 3). This "genome obesity" is the likely result of a steady accumulation of transposable elements and an inability to discard them, as found in smaller flowering plant genomes ${ }^{8,40}$. Importantly, Ceratopteris had a very low diversity of recent LTR RT exemplars when compared to other large-genome species such as maize or Picea. While this finding could be indicative of low LTR RT richness and high abundance, given that the counts of the LTR RTs were considerably higher in Ceratopteris compared to the other genomes, it is also possible that we are unable to identify the majority of full-length LTR RTs due to low scaffold contiguity with the CFern $v 1.1$ assembly. The BAC.SubSample assembly and read-based analyses provide a more accurate representation of the general repeat composition of Ceratopteris; however, these results provide a much smaller representation of the genome and are limited in their resolution. Clearly, long-read technology will 
be necessary to overcome and fully analyze a genome of this size, as short-read sequencing simply cannot span and assemble the repetitive structures found in Ceratopteris. However, expense must be taken into account in any sequencing project, and here the deep sequencing of an $11.25 \mathrm{Gbp}$ genome using long-read technology would take much longer and cost much more than similar sequencing with short reads.

This study provides a major stepping-stone in the understanding of plant evolutionary genomics by providing the first homosporous fern reference genome, as well as unique insights into the processes underlying the formation of these massive genomes. Future efforts should focus on long-read technology to provide a complete assembly of multiple homosporous ferns-thus permitting more extensive comparisons of genome evolution and synteny across green plants.

\section{Methods}

Tissue samples. Ceratopteris richardii (Pteridaceae) is a fast-growing tropical fern, used globally in research laboratories as well as in K-12 and undergraduate biology courses for studying alternation of generations in plants. Inbred lines and single-gene mutants are commercially available and readily produced. For this study, spores from the Hn-n inbred line were kindly donated by Dr. Leslie Hickok (University of Tennessee). The spores were germinated on nutrient media ${ }^{77,78}$ and grown following the recommended conditions in the C-Fern Manual (www.c-fern.org). We isolated the germinated gametophytes to individual petri dishes and growth media. Given that $C$. richardii is homosporous, the gametophytes are typically bisexual and produce both antheridia and archegonia. By isolating the gametophytes prior to sexual maturity, we ensured that any sporophytes that did develop were a product of gametes from a single gametophyte and thus completely homozygous (doubled haploid).

Library construction and sequencing. We extracted genomic DNA (gDNA) from Ceratopteris using a modified CTAB protocol ${ }^{79}$ and quality checked and quantified the gDNA using a Qubit fluorometer (Invitrogen, Carlsbad, CA, USA) and NanoDrop spectrophotometer (Thermo Fisher Scientific, Waltham, MA, USA). Genomic short-read library preparation and sequencing for Ceratopteris were completed by the University of Florida's Interdisciplinary Center for Biotechnology Research (UF ICBR). The gDNA was fragmented and size-selected for $\sim 300$ base pair (bp) inserts, and the sequencing of 150 bp paired-end (PE) reads was conducted on two runs of the Illumina NextSeq platform (Illumina, San Diego, CA, USA). Mate-pair (MP) libraries (125 bp PE, 8-10 Kbp inserts) were prepared and sequenced at the Duke Genome Sequencing and Analysis Core on two lanes of Illumina HiSeq 2000 (Illumina, San Diego, CA, USA).

We also subsampled the Ceratopteris genome with long-read technology to avoid the assembly biases inherent in short-read technology by sequencing BAC clones (Plate CR_Ba \#624, Green Plant BAC Library Project, provided by Clemson University Genomics Institute). We selected 34 Ceratopteris $\mathrm{Hn}-\mathrm{n}$ BAC clones to be grown, pooled, purified, and sequenced using the RS II platform (Pacific Bioscience, Menlo Park, CA, USA) at the Arizona Genomics Institute. The reads were cleaned and de novo assembled using the Hierarchical Genome Assembly Process (HGAP) in the SMRT Analysis software package (Pacific Biosciences, Menlo Park, CA, USA) to produce the BAC.SubSample assembly.

Long-read technology was also used to acquire a high-confidence set of gene models from sporophyte tissue. We extracted total RNA from sexually mature leaf tissue using the RNeasy Plant Mini kit (Qiagen, Hilden, Germany). The total RNA was size-selected for $0.8-2,2-3,3-5$, and $>5 \mathrm{Kbp}$ with the SageELF (Sage Science, Beverly, MA, USA) at the UF ICBR. The libraries were prepared following the SMRTbell library protocol, and each library was sequenced on three PacBio SMRT cells (Pacific Bioscience, Menlo Park, CA, USA) at the UF ICBR.

Genome assembly. The raw genomic PE reads were trimmed of adapters and then quality-filtered with Trimmomatic ${ }^{80}$, while the raw MP reads were trimmed of adapters and separated into MP, PE, and unknown reads with $\mathrm{NxTrim}^{81}$. All libraries were quality-checked before and after cleaning with FastQC (www.bioinformatics.babraham.ac.uk/projects/fastqc/). We divided the cleaned PE reads into 24 -mers with Jellyfish ${ }^{82}$ and plotted their frequencies with $\mathrm{KAT}^{83}$ to assess environmental contamination, organellar genome content, nuclear genome size, and repeat content.

The PE reads were assembled using Meraculous $2^{84}$ and a k-mer size of 61 based on the results of KmerGenie ${ }^{85}$ to produce assembly CFern v1.0. The scaffolds from the CFern $v 1.0$ assembly were further scaffolded with the MP reads using the SSPACE assembler ${ }^{86}$ to produce the genome assembly CFern $v 1.1$. To compare the content of CFern v1.1 with the overall content of the cleaned reads, we divided the assembly into 24-mers with Jellyfish ${ }^{82}$ and compared the resulting frequencies to those of the cleaned PE reads using the compare feature of $\mathrm{KAT}^{83}$. For subsequent analyses, only scaffolds over $10 \mathrm{Kbp}$ were used (CFern $1.1 \mathrm{~A})$.

Transcriptome assembly. We cleaned and processed the long reads following the IsoSeq protocol ${ }^{54}$ in which the circular consensus sequences (CCS) were acquired from the raw reads and then classified and clustered. Only full-length, high-quality (accuracy $>=99 \%$ ), polished sequences (IsoSeq.HQ) were used for analysis following the Iterative Clustering and Error correction (ICE)/Quiver algorithm. The IsoSeq. HQ sequences were further collapsed into unique isoforms and genes using both genome-based and sequence-based protocols (see below).

For the genome-based method, the IsoSeq.HQ sequences were mapped to the CFern $v 1.1 \mathrm{~A}$ assembly using GMAP (parameters: -f samse $-\mathrm{n} 0-\mathrm{z}$ sense_force) ${ }^{87}$. The sam file output was sorted (parameters: $-\mathrm{k} 3,3-\mathrm{k} 4,4 \mathrm{n}$ ), and transcripts were collapsed together (collapse_isoforms_by_sam.py, https://github.com/Magdoll/cDNA_ Cupcake $)^{54}$. We used both $98 \%$ coverage and $98 \%$ identity as our full-length mapping cutoff and then searched for incomplete genes with $50 \%$ coverage and $98 \%$ identity. 
Due to the fragmented state of the CFern $v 1.1 A$ assembly, many transcripts did not map. Thus, we also used CD-Hit v4.6.4 (parameters: -c 0.99 -G 0 -aL 0.90 -AL 100 -aS $0.99-$ AS 30$)^{88}$ to cluster and collapse highly similar transcripts into putative isoforms without a reference genome. We then used those sequences with the Coding Genome reconstruction tool ${ }^{89}$ for genome-free isoform collapse and gene identification. This pipeline divided the sequences into 30-mers and then grouped those kmers into clusters based on pairwise distances. De Bruijn graphs of the sequences for each cluster were then used to resolve sequencing errors and alternative splicing events and output putative genes. Due to the high accuracy, full-length, de novo nature of IsoSeq and subsequent cleaning protocols, these genes served as our reference gene models for Ceratopteris (referred to as UniCFernModels).

Polyploidy. The UniCFernModels data set was used in the DupPipe pipeline ${ }^{90,91}$ to estimate the relative age of gene duplications. DupPipe finds duplicate gene pairs and then estimates the divergence of these genes using the number of substitutions per synonymous site $\left(K_{S}\right)$. The frequency of duplicate genes corresponding to a given level of divergence, as a substitute for timing, was plotted as a histogram, and peaks were inferred to represent synchronous gene duplications, indicative of ancient polyploidy events ${ }^{90,92}$. Genes from two other ferns, Equisetum giganteum ${ }^{57}$ and Azolla filiculoides ${ }^{19}$, were similarly analyzed and plotted for comparison. To reduce the subjectivity of smoothing based on varying bin sizes, we analyzed the $K_{S}$ values of these three ferns using the SiZer (Significance of Zero Crossings of the Derivative) ${ }^{93}$ package in R v3.4.2 ${ }^{94}$. This analysis determines whether an increase or decrease in a scatterplot or histogram is significant at $\alpha=0.05$ and plots the changes along the original $x$-axis with blue coloration indicating a significant increase, red a significant decrease, purple insignificance, and gray too few data points to determine.

To determine whether the three ferns examined here (Ceratopteris, Equisetum, and Azolla), spanning over 400 million years since their most recent common ancestor ${ }^{70}$, share any ancient polyploidy events, we clustered the predicted proteins of Ceratopteris, Equisetum, Azolla, Amborella, Selaginella, and Physcomitrella into orthogroups using OrthoFinder ${ }^{95}$. Only orthogroups with gene representatives from all six species were retained. The protein sequences of each orthogroup were aligned with $\mathrm{MAFFT}^{96}$, and the alignments were converted to nucleotide alignments using the pxaa2cdn tool in Phyx ${ }^{97}$. The alignments were stripped of highly ambiguous $(>90 \%$ missing data) columns, and gene trees were produced with RAxML using 100 rapid bootstrap searches and the GTRGAMMA model of evolution ${ }^{98}$. These gene family trees were entered into the Multi-tAxon Paleopolyploidy Search (MAPS) package ${ }^{58}$. This package first filters all of the gene family trees for subtrees that match the known species tree [here (Physcomitrella, (Selaginella, (Amborella, (Equisetum, (Ceratopteris, Azolla)))))]. It then counts the number of subtrees with gene duplications at a specific node in the species tree relative to the number of available subtrees. A node with a high proportion of gene duplications is presumed to have a shared polyploidy event.

We also used a cytogenetic approach to assess more recent WGD. We conducted fluorescent in situ hybridization (FISH) using the previously described BAC clones as probes following Chester et al. ${ }^{99}$ and Chamala et al. ${ }^{100}$. To produce the probes, the BAC DNA was extracted from the Escherichia coli culture and amplified by rolling circle amplification $(\mathrm{RCA})^{101}$. The RCA product was labeled by nick translation with Cy5-dUTP and purified with a QIAquick Nucleotide Removal kit (Qiagen, Venlo, Netherlands).

Root tips for chromosome preparations were collected in the mornings and immediately treated with pressurized nitrous oxide for 1 hour before being fixed in 3:1 ethanol (EtOH): glacial acetic acid overnight at room temperature and transferred to $70 \% \mathrm{EtOH}$ at $-20^{\circ} \mathrm{C}$ for long-term storage. The root tips were then treated and chromosome spreads prepared to produce slides for in situ hybridization with the fluorescently labeled probes ${ }^{99}$. The BAC FISH images were taken on an AxioImager M2 microscope with an AxioCam MR camera (Carl Zeiss AG, Oberkochen, Germany).

Repeat characterization. We took both structural- and homology-based approaches to repeat characterization following Campbell et al. ${ }^{102}$. As long terminal repeat retrotransposons (LTR RTs) comprise a sizable proportion of most plant genomes, a variety of tools was used to characterize these repeats in the CFern v1.1A assembly. Recent LTR RTs were collected based on 90\% LTR similarity using LTRharvest (parameters: - minlenltr 100 -maxlenltr 6000 -mindistltr 1500 -maxdistltr 25000 -mintsd 5 -maxtsd 5 -motif tgca -similar 90 -vic 10) $)^{103}$ from the GenomeTools package ${ }^{104}$. LTRdigest was then used to find elements with poly purine tracts (PPT) or primer binding sites (PBS) using the Genomic tRNA database ${ }^{105}$. Those elements were identified and further filtered for false positive elements by removing gappy elements $(>50 \mathrm{Ns})$, recent gene duplications where the flanking regions of the LTRs are alignable, and nested RT insertions using custom scripts. LTR RTs with nested DNA transposons were also identified by searching DNA transposase protein sequences with BLASTx ${ }^{106}$. LTR RT exemplars were then identified based on $80 \%$ identity and $90 \%$ coverage from the filtered elements based on the internal sequences of the LTR RTs and then based on the LTR sequences. Older LTR RTs were similarly collected but with $75 \%$ similarity among the LTR sequences and lacking the TGCA motif. To exclude more recent LTR RTs from the older LTR RT library, the younger LTR RT exemplars were used to mask and exclude elements found in the older LTR RT library with RepeatMasker ${ }^{107}$. The two LTR RT libraries were combined (allLTR.lib) and used as the reference library to mask the CFern $v 1.1 A$ assembly with RepeatMasker ${ }^{107}$.

The unmasked remainder of the assembly was inputted into RepeatModeler to identify repeat families de novo ${ }^{108}$. The RepeatModeler library and LTR RT library were combined, and unidentified repeats were searched against a transposase database ${ }^{107,109}$ using BLASTx and identified to superfamily when possible ${ }^{106}$. To ensure that fragmented plant genes were not included in the final repeat library, we queried all of our repeats with the SwissProt plant protein ${ }^{110}$ and NCBI RefSeq plant protein databases using BLASTx ${ }^{106}$. With our clean, final repeat library, we used RepeatMasker to quantify the repeat elements throughout CFern v1.1A.

To make direct comparisons with other plant genome assemblies of varying sizes, qualities, and lineages, we followed the same repeat annotation protocol for the genomes of Amborella trichopoda ${ }^{16}$, a monocot (Zea mays $)^{60}$, a liverwort (Marchantia polymorpha $)^{61}$, a lycophyte (Selaginella moellendorffi $)^{62}$, a conifer (Picea abies) ${ }^{8}$, 
and a moss (Physcomitrella patens) ${ }^{63}$. We also ran the same protocol on the BAC.SubSample assembly. To remove assembly biases, we used RepeatExplorer $2^{111}$ on cleaned reads for the above-mentioned taxa, with the exception of Selaginella which only used Sanger sequencing. Raw reads were downloaded from the NCBI Sequence Read Archive and EMBL European Nucleotide Archive, cleaned and reduced to 0.5X coverage of their respective genomes, then run in RepeatExplorer2 ${ }^{111}$ via the Elixir CZ Galaxy portal under default parameters against the Viridiplantae version 3.0 transposable element protein domain database.

Dating repeat insertion events. We used the highly accurate but conservative LTR_Retriever package ${ }^{65}$ to identify full-length LTR RTs and date their insertion using both the CFern v1.1A and BAC.SubSample assemblies. We provided candidate LTR RTs from LTR_harvest and LTR_finder using a 90\% similarity minimum threshold between LTRs and the presence of the TGCA motifs. The candidate LTR RTs were filtered, removing non-LTR $\mathrm{RT}$ repeat elements or those with large amounts of tandem repeats or gaps. Especially in fragmented genome assemblies, such as the CFern $v 1.1 A$, these requirements hugely reduce the number of LTR RT candidates but ensure that only full-length LTR RTs are analyzed. Following filtering, the long terminal repeat regions of each transposable element were aligned, and the Jukes-Cantor model was used to estimate the divergence time of the two LTR regions. We used a mutation rate of $6.5 \times 10^{-9}$ per site per year to estimate the years since insertion ${ }^{16}$. This mutation rate is half that of rice $^{36}$ and is a general estimate; therefore, the insertion times should only be used in reference to the relative timing of insertion, rather than as exact dates.

\section{Data availability}

All of the raw reads and associated genomic and transcriptomic assemblies can be found in the NCBI BioProjects under PRJNA511033. All of the tissue used for sequencing came from one doubled haploid genotype (Voucher: M. Whitten \#5841, University of Florida Herbarium, FLAS).

Received: 2 January 2019; Accepted: 4 November 2019;

Published online: 03 December 2019

\section{References}

1. Lughadha, E. N. et al. Counting counts: Revised estimates of numbers of accepted species of flowering plants, seed plants, vascular plants and land plants with a review of other recent estimates. Phytotaxa 272, 82-88 (2016).

2. Bennett, M. D. \& Leitch, I. J. Plant DNA C-values database (release 6.0, Dec. 2012). WWW Doc. URL http//data.kew.org/cvalues/. [accessed 14 Oct. 2014] (2012).

3. Rice, A. et al. The Chromosome Counts Database (CCDB) - a community resource of plant chromosome numbers. New Phytol. 206, 19-26 (2015).

4. Greilhuber, J. et al. Smallest angiosperm genomes found in Lentibulariaceae, with chromosomes of bacterial size. Plant Biol. 8, 770-777 (2006).

5. Pellicer, J., Fay, M. F. \& Leitch, I. J. The largest eukaryotic genome of them all? Bot. J. Linn. Soc. 164, 10-15 (2010).

6. Ghatak, J. Biosystematic survey of pteridophytes from Shevaroy Hills, south India. Nucleus 20, 105-108 (1977).

7. Bennett, M. D. Plant genome values: How much do we know? Proc. Natl. Acad. Sci. 95, 2011-2016 (1998).

8. Nystedt, B. et al. The Norway spruce genome sequence and conifer genome evolution. Nature 497, 579-584 (2013).

9. Zimin, A. et al. Sequencing and assembly of the 22-gb loblolly pine genome. Genetics 196, 875-90 (2014).

10. Birol, I. et al. Assembling the $20 \mathrm{~Gb}$ white spruce (Picea glauca) genome from whole-genome shotgun sequencing data. Bioinforma. https://doi.org/10.1093/bioinformatics/btt178 (2013).

11. Stebbins, G. L. Jr The significance of polyploidy in plant evolution. Am. Nat. 74, 54-66 (1940).

12. Grant, V. Plant Speciation (Columbia University Press, 1981).

13. Jiao, Y. et al. A genome triplication associated with early diversification of the core eudicots. Genome Biol. 13, R3 (2012).

14. Tang, H., Bowers, J. E., Wang, X. \& Paterson, A. H. Angiosperm genome comparisons reveal early polyploidy in the monocot lineage. Proc. Natl. Acad. Sci. 107, 472-477 (2010).

15. Jiao, Y. et al. Ancestral polyploidy in seed plants and angiosperms. Nature 473, 97-100 (2011).

16. Amborella Genome Project. The Amborella Genome and the Evolution of Flowering Plants. Science (80-.), 342, (2013).

17. Landis, J. B. et al. Impact of whole-genome duplication events on diversification rates in angiosperms. Am. J. Bot. (2018).

18. Carretero-Paulet, L. et al. High Gene Family Turnover Rates and Gene Space Adaptation in the Compact Genome of the Carnivorous Plant Utricularia gibba. Mol. Biol. Evol. 32, 1284-1295 (2015)

19. Li, F.-W. et al. Fern genomes elucidate land plant evolution and cyanobacterial symbioses. Nat. plants, 1 (2018).

20. Klekowski, E. \& Baker, H. Evolutionary Significance of Polyploidy in the Pteridophyta. Science (80-.). 153, 305-307 (1966).

21. Haufler, C. H. Electrophoresis is Modifying Our Concepts of Evolution in Homosporous Pteridophytes. Am. J. Bot. 74, 953-966 (1987).

22. Wagner, W. H. \& Wagner, F. S. Polyploidy in pteridophytes. In Polyploidy 199-214 (Springer, 1980).

23. Klekowski, E. Genetical features of ferns as contrasted with seed plants. Ann. Missouri Bot. Gard. 59, 138-151 (1972).

24. Haufler, C. H. et al. Sex and the single gametophyte: Revising the homosporous vascular plant life cycle in light of contemporary research. Bioscience 66, 928-937 (2016).

25. Soltis, D. E. Genetic evidence for diploidy in Equisetum. Am. J. Bot., 908-913 (1986).

26. Haufler, C. H. \& Soltis, D. E. Evolutionary Significance of Polyploidy in the Pteridophyta., 83, 4389-4393 (1986).

27. Soltis, P. S. \& Soltis, D. E. Electrophoretic evidence for genetic diploidy in Psilotum nudum. Am. J. Bot., 1667-1671 (1988),

28. Pichersky, E., Soltis, D. \& Soltis, P. Defective chlorophyll a/b-binding protein genes in the genome of a homosporous fern. Proc. Natl. Acad. Sci. 87, 195-199 (1990).

29. Soltis, D. E. \& Soltis, P. S. The distribution of selfing rates in homosporous ferns. Am. J. Bot. (1992).

30. Nakazato, T., Jung, M.-K., Housworth, E. A., Rieseberg, L. H. \& Gastony, G. J. Genetic map-based analysis of genome structure in the homosporous fern Ceratopteris richardii. Genetics 173, 1585-97 (2006).

31. Barker, M. S. Evolutionary Genomic Analyses of Ferns Reveal that High Chromosome Numbers are a Product of High Retention and Fewer Rounds of Polyploidy Relative to Angiosperms. Am. Fern J., 99, 136-141 CR-Copyright \&\#169; 2009 American Fern (2009).

32. Wood, T. E. et al. The frequency of polyploid speciation in vascular plants. Proc. Natl. Acad. Sci. 106, 13875-13879 (2009).

33. Nakazato, T., Barker, M. S., Rieseberg, L. H. \& Gastony, G. J. Evolution of the nuclear genome of ferns and lycophytes. In Biology and evolution of ferns and lycophytes (Cambridge University Press, 2008). 
34. Sessa, E. B. \& Der, J. P. Evolutionary genomics of ferns and lycophytes. In Advances in Botanical Research 78, 215-254 (Elsevier, 2016).

35. Piegu, B. et al. Doubling genome size without polyploidization: Dynamics of retrotransposition-driven genomic expansions in Oryza australiensis, a wild relative of rice. Genome Res. 16, 1262-1269 (2006).

36. Ma, J. \& Bennetzen, J. L. Rapid recent growth and divergence of rice nuclear genomes. Proc. Natl. Acad. Sci. USA 101, 12404 LP-12410 (2004).

37. Vitte, C. \& Bennetzen, J. L. Analysis of retrotransposon structural diversity uncovers properties and propensities in angiosperm genome evolution. Proc. Natl. Acad. Sci. 103, 17638-17643 (2006).

38. Estep, M. C., DeBarry, J. D. \& Bennetzen, J. L. The dynamics of LTR retrotransposon accumulation across 25 million years of panicoid grass evolution. Heredity (Edinb). 110, 194-204 (2013).

39. Bennetzen, J. L. \& Wang, H. The contributions of transposable elements to the structure, function, and evolution of plant genomes. Annu. Rev. Plant Biol. 65, 505-530 (2014).

40. Devos, K. M., Brown, J. K. M. \& Bennetzen, J. L. Genome Size Reduction through Illegitimate Recombination Counteracts Genome Expansion in Arabidopsis. Genome Res. 12, 1075-1079 (2002).

41. Hawkins, J. S., Kim, H., Nason, J. D., Wing, R. A. \& Wendel, J. F. Differential lineage-specific amplification of transposable elements is responsible for genome size variation in Gossypium. Genome Res. 16, 1252-1261 (2006).

42. De La Torre, A. R. et al. Insights into conifer giga-genomes. Plant Physiol. 166, 1724-32 (2014).

43. Soltis, D. E. \& Soltis, P. S. Polyploidy and Breeding Systems in Homosporous Pteridophyta: A Reevaluation. Am. Nat. 130, 219-232 (1987).

44. Sessa, E. B. et al. Between Two Fern Genomes. Gigascience, 3, (2014)

45. Durand, L. Z. \& Goldstein, G. Photosynthesis, photoinhibition, and nitrogen use efficiency in native and invasive tree ferns in Hawaii. Oecologia 126, 345-354 (2001).

46. Ellwood, M. D. F. \& Foster, Wa Doubling the estimate of invertebrate biomass in a rainforest canopy. Nature 429, 549-51 (2004).

47. Fayle, T. M., Chung, A. Y. C., Dumbrell, A. J., Eggleton, P. \& Foster, W. A. The Effect of Rain Forest Canopy Architecture on the Distribution of Epiphytic Ferns (Asplenium spp.) in Sabah, Malaysia. Biotropica 41, 676-681 (2009).

48. Paul, B. et al. Azolla domestication towards a biobased economy? New Phytol. 202, 1069-1082 (2014).

49. Shukla, A. K. et al. Expression of an insecticidal fern protein in cotton protects against whitefly. Nat. Biotechnol. 34, 1046 (2016).

50. PPG I. A community derived classification for extant lycophytes and ferns. J. Syst. Evol. 54, 563-603 (2016).

51. Pryer, K. M., Schneider, H., Zimmer, E. A. \& Ann Banks, J. Deciding among green plants for whole genome studies. Trends Plant Sci. 7, 550-554 (2002).

52. Soltis, P. S. \& Soltis, D. E. A conifer genome spruces up plant phylogenomics. Genome Biol. 14, 122 (2013).

53. Warren, W. C. et al. Genome analysis of the platypus reveals unique signatures of evolution. Nature 453, 175-183 (2008).

54. Gordon, S. P. et al. Widespread polycistronic transcripts in fungi revealed by single-molecule mRNA sequencing. PLoS One 10, e0132628 (2015).

55. Simão, F. A., Waterhouse, R. M., Ioannidis, P., Kriventseva, E. V. \& Zdobnov, E. M. BUSCO: assessing genome assembly and annotation completeness with single-copy orthologs. Bioinformatics 31, 3210-3212 (2015).

56. Cui, L. et al. Widespread genome duplications throughout the history of flowering plants. Genome Res. 16, 738-749 (2006).

57. Vanneste, K., Sterck, L., Myburg, Z., Van de Peer, Y. \& Mizrachi, E. Horsetails Are Ancient Polyploids: Evidence from Equisetum giganteum. Plant Cell, 1-13 https://doi.org/10.1105/tpc.15.00157 (2015).

58. Li, Z. et al. Early genome duplications in conifers and other seed plants. Sci. Adv., 1, (2015).

59. Lim, K. A. R. Y., Matyaksek, R., Kovarik, A. \& Leitch, A. R. Genome evolution in allotetraploid. Nicotiana. Biol. J. Linn. Soc. 82, 599-606 (2004)

60. Hirsch, C. et al. Draft assembly of elite inbred line PH207 provides insights into genomic and transcriptome diversity in maize. Plant Cell tpc-00353 (2016).

61. Bowman, J. L. et al. Insights into land plant evolution garnered from the Marchantia polymorpha genome. Cell 171, 287-304 (2017).

62. Banks, J. A. et al. The Selaginella Genome Identifies Genetic Changes Associated with the Evolution of Vascular. Plants. Sci. 332, 960-963 (2011).

63. Lang, D. et al. The Physcomitrella patens chromosome-scale assembly reveals moss genome structure and evolution. Plant J. 93, 515-533 (2018)

64. Wolf, P. G. et al. An exploration into fern genome space. Genome Biol. Evol. https://doi.org/10.1093/gbe/evv163 (2015).

65. Ou, S. \& Jiang, N. LTR_retriever: A Highly Accurate and Sensitive Program for Identification of Long Terminal Repeat Retrotransposons. Plant Physiol. 176, 1410-1422 (2018).

66. Smith, A. R. et al. A classification for extant ferns. Taxon 55, 705-731 (2006).

67. Morris, J. L. et al. The timescale of early land plant evolution. Proc. Natl. Acad. Sci. 115, E2274-E2283 (2018).

68. Skog, J. E. \& Banks, H. P. Ibyka amphikoma, gen. et sp. n., a new protoarticulate precursor from the late Middle Devonian of New York State. Am. J. Bot. 60, 366-380 (1973).

69. Schuettpelz, E. \& Pryer, K. M. Fern phylogeny inferred from 400 leptosporangiate species and three plastid genes. Taxon 56, 1037 (2007).

70. Testo, W. \& Sundue, M. A 4000-species dataset provides new insight into the evolution of ferns. Mol. Phylogenet. Evol. 105, 200-211 (2016).

71. Zhang, R. et al. Dating Whole Genome Duplication in Ceratopteris thalictroides and Potential Adaptive Values of Retained Gene Duplicates. Int. J. Mol. Sci. 20, 1926 (2019).

72. De La Torre, A. R., Li, Z., Van de Peer, Y. \& Ingvarsson, P. K. Contrasting rates of molecular evolution and patterns of selection among gymnosperms and flowering plants. Mol. Biol. Evol. 34, 1363-1377 (2017).

73. Bromham, L., Hua, X., Lanfear, R. \& Cowman, P. F. Exploring the relationships between mutation rates, life history, genome size, environment, and species richness in flowering plants. Am. Nat. 185, 507-524 (2015).

74. Haufler, C. H. Homospory 2002: An Odyssey of progress in pteridophyte genetics and evolutionary biology: Ferns and other homosporous vascular plants have highly polyploid chromosome numbers, but they express traits following diploid models and, although capable of extre. AIBS Bull. 52, 1081-1093 (2002).

75. Haufler, C. H. Ever since Klekowski: testing a set of radical hypotheses revives the genetics of ferns and lycophytes. Am. J. Bot. 101, 2036-2042 (2014)

76. Clark, J. et al. Genome evolution of ferns: evidence for relative stasis of genome size across the fern phylogeny. New Phytol. 210, 1072-1082 (2016).

77. Bold, H. C. Morphology of Plants (Harper and Row, 1957)

78. Nitsch, J. P. Growth and development in vitro of excised ovaries. Am. J. Bot., 566-577 (1951).

79. Doyle, J. \& Doyle, J. L. Genomic plant DNA preparation from fresh tissue-CTAB method. Phytochem Bull 19, 11-15 (1987).

80. Bolger, A. M., Lohse, M. \& Usadel, B. Trimmomatic: a flexible trimmer for Illumina sequence data. Bioinformatics 30, 2114-2120 (2014).

81. O’Connell, J. et al. NxTrim: optimized trimming of Illumina mate pair reads. Bioinformatics 31, 2035-2037 (2015). 
82. Marçais, G. \& Kingsford, C. A fast, lock-free approach for efficient parallel counting of occurrences of k-mers. Bioinformatics 27, 764-770 (2011).

83. Mapleson, D., Garcia Accinelli, G., Kettleborough, G., Wright, J. \& Clavijo, B. J. KAT: a K-mer analysis toolkit to quality control NGS datasets and genome assemblies. Bioinformatics 33, 574-576 (2016).

84. Chapman, J. A., Ho, I. Y., Goltsman, E. \& Rokhsar, D. S. Meraculous2: fast accurate short-read assembly of large polymorphic genomes. arXiv Prepr. arXiv1608.01031 (2016).

85. Chikhi, R. \& Medvedev, P. Informed and automated k-mer size selection for genome assembly. Bioinformatics 30, 31-37 (2014).

86. Boetzer, M., Henkel, C. V., Jansen, H. J., Butler, D. \& Pirovano, W. Scaffolding pre-assembled contigs using SSPACE. Bioinformatics 27, 578-579 (2010).

87. Wu, T. D. \& Watanabe, C. K. GMAP: a genomic mapping and alignment program for mRNA and EST sequences. Bioinformatics 21, 1859-1875 (2005).

88. Fu, L., Niu, B., Zhu, Z., Wu, S. \& Li, W. CD-HIT: accelerated for clustering the next-generation sequencing data. Bioinformatics 28, 3150-3152 (2012).

89. Workman, R. E. et al. Single-molecule, full-length transcript sequencing provides insight into the extreme metabolism of the rubythroated hummingbird Archilochus colubris. Gigascience 7, giy009 (2018).

90. Barker, M. S. et al. Multiple paleopolyploidizations during the evolution of the Compositae reveal parallel patterns of duplicate gene retention after millions of years. Mol. Biol. Evol. 25, 2445-2455 (2008).

91. Barker, M. S. et al. EvoPipes. net: bioinformatic tools for ecological and evolutionary genomics. Evol. Bioinforma. 6, EBO-S5861 (2010).

92. Lynch, M. \& Conery, J. S. The Evolutionary Fate and Consequences of Duplicate. Genes. Sci. 290, 1151-1155 (2000).

93. Chaudhuri, P. \& Marron, J. S. SiZer for exploration of structures in curves. J. Am. Stat. Assoc. 94, 807-823 (1999).

94. R Core Team. R: A language and environment for statistical computing. (2013).

95. Emms, D. M. \& Kelly, S. OrthoFinder: solving fundamental biases in whole genome comparisons dramatically improves orthogroup inference accuracy. Genome Biol. 16, 157 (2015).

96. Katoh, K. \& Standley, D. M. MAFFT multiple sequence alignment software version 7: improvements in performance and usability. Mol. Biol. Evol. 30, 772-780 (2013).

97. Brown, J. W., Walker, J. F. \& Smith, S. A. Phyx: phylogenetic tools for unix. Bioinformatics 33, 1886-1888 (2017).

98. Stamatakis, A. RAxML version 8: a tool for phylogenetic analysis and post-analysis of large phylogenies. Bioinforma. 30, 1312-1313 (2014).

99. Chester, M. et al. Extensive chromosomal variation in a recently formed natural allopolyploid species, Tragopogon miscellus (Asteraceae). Proc. Natl. Acad. Sci. 109, 1176-1181 (2012).

100. Chamala, S. et al. Assembly and validation of the genome of the nonmodel basal angiosperm Amborella. Science 342, 1516-7 (2013).

101. Berr, A. \& Schubert, I. Direct labelling of BAC DNA by rolling circle amplification. Plant J. 45, 857-862 (2006).

102. Campbell, M. S. et al. MAKER-P: a tool kit for the rapid creation, management, and quality control of plant genome annotations. Plant Physiol. 164, 513-524 (2014).

103. Ellinghaus, D., Kurtz, S. \& Willhoeft, U. LTRharvest, an efficient and flexible software for de novo detection of LTR retrotransposons. BMC Bioinformatics 9, 18 (2008).

104. Gremme, G., Steinbiss, S. \& Kurtz, S. GenomeTools: a comprehensive software library for efficient processing of structured genome annotations. IEEE/ACM Trans. Comput. Biol. Bioinforma. 10, 645-656 (2013).

105. Chan, P. P. \& Lowe, T. M. GtRNAdb 2.0: an expanded database of transfer RNA genes identified in complete and draft genomes. Nucleic Acids Res. 44, D184-D189 (2015)

106. Camacho, C. et al. BLAST+: architecture and applications. BMC Bioinformatics 10, 421 (2009).

107. Smith, A. F. A., Hubley, R. \& Green, P. RepeatMasker Open-4.0. 2013-2015.

108. Smit, A. F. A. \& Hubley, R. RepeatModeler Open-1.0. Available fom http//www.repeatmasker.org (2008)

109. Kennedy, R. C., Unger, M. F., Christley, S., Collins, F. H. \& Madey, G. R. An automated homology-based approach for identifying transposable elements. BMC Bioinformatics 12, 130 (2011).

110. Schneider, M. et al. The UniProtKB/Swiss-Prot knowledgebase and its Plant Proteome Annotation Program. J. Proteomics 72, 567-573 (2009).

111. Novák, P., Neumann, P., Pech, J., Steinhaisl, J. \& Macas, J. RepeatExplorer: a Galaxy-based web server for genome-wide characterization of eukaryotic repetitive elements from next-generation sequence reads. Bioinformatics 29, 792-793 (2013).

\section{Acknowledgements}

This research was funded by a University of Florida Genetics Institute Seed Award. DBM was supported by iDigBio (NSF-DBI-1547229). The authors thank Dr. Matias Kirst for his edits and advice during revisions of the manuscript.

\section{Author contributions}

D.B.M., P.S.S. and D.E.S. designed the study with advice from E.B.S., P.G.W. and W.B.B. K.H. optimized the BACFISH protocol for Ceratopteris. D.B.M. carried out the study, analyzed the data, and wrote the manuscript. All authors reviewed and edited the manuscript.

\section{Competing interests}

The authors declare no competing interests.

\section{Additional information}

Correspondence and requests for materials should be addressed to D.B.M.

Reprints and permissions information is available at www.nature.com/reprints.

Publisher's note Springer Nature remains neutral with regard to jurisdictional claims in published maps and institutional affiliations. 
(c) (i) Open Access This article is licensed under a Creative Commons Attribution 4.0 International License, which permits use, sharing, adaptation, distribution and reproduction in any medium or format, as long as you give appropriate credit to the original author(s) and the source, provide a link to the Creative Commons license, and indicate if changes were made. The images or other third party material in this article are included in the article's Creative Commons license, unless indicated otherwise in a credit line to the material. If material is not included in the article's Creative Commons license and your intended use is not permitted by statutory regulation or exceeds the permitted use, you will need to obtain permission directly from the copyright holder. To view a copy of this license, visit http://creativecommons.org/licenses/by/4.0/.

(C) The Author(s) 2019 